\title{
Clinical Utility of Silk-Elastin Sponge in Patients with Chronic and Acute Skin Ulcers: Study Protocol of a Multicenter Clinical Trial
}

\author{
Kazuo Noda (D) - Yasuhiro Katayama · Eiichi Sawaragi · \\ Michiharu Sakamoto · Hiroki Yamanaka · Itaru Tsuge • \\ Motoki Katsube · Naoki Morimoto
}

Received: October 6, 2021 / Accepted: November 18, 2021 / Published online: November 30, 2021

(C) The Author(s) 2021

\section{ABSTRACT}

Introduction: Not only chronic but also some acute wounds have a risk of infection and become unhealed wounds. Silk-elastin sponge has been developed to treat chronic wounds that are susceptible to infection. Preclinical and clinical studies suggested that silk-elastin sponge is safe for humans and can promote granulation tissue formation by reducing bacterial growth in chronic wounds. The central aim of this trial is to evaluate the clinical utility and safety of silk-elastin sponge for the treatment of chronic and acute skin ulcers.

Methods: This study is a prospective, multicenter, single-arm, uncontrolled clinical trial. In this study, 20 patients with chronic ulcers and five with an acute one will be included; patients with wound infection will be excluded. Silkelastin sponges are applied and covered with a dressing for 14 days.

K. Noda $(\bowtie) \cdot$ Y. Katayama · E. Sawaragi ·

M. Sakamoto $\cdot$ H. Yamanaka $\cdot$ I. Tsuge $\cdot$ M. Katsube - N. Morimoto

Department of Plastic and Reconstructive Surgery, Graduate School of Medicine, Kyoto University, 54 Kawahara-cho, Shogoin, Sakyo-ku, Kyoto 606-8507, Japan

e-mail: knoda@kuhp.kyoto-u.ac.jp

K. Noda

Department of Plastic and Reconstructive Surgery,

Kurashiki Central Hospital, Kurashiki, Japan
Planned Outcomes: The primary endpoint is the frequency of patients with chronic wounds in whom the investigator confirms the formation of a healthy wound bed at 14 days after the initial application of the study device. In addition, safety for acute wounds and handiness of the study device will be assessed.

Trial registration number: jRCT2052210072.

Keywords: Study protocol; Wound healing; Silk-elastin

\section{Key Summary Points}

The central aim of this trial is to evaluate the clinical utility and safety of silk-elastin sponge for the treatment of skin ulcers.

This study is a prospective, multicenter, single-arm, uncontrolled clinical trial.

This trial intends to treat not only chronic but also acute wounds.

This is not a large-scale study. 


\section{INTRODUCTION}

\section{Background}

Ulcers or wounds can be divided into two groups: acute and chronic [1]. Acute wounds are formed by traumatic damage such as injury, surgery, and thermal or chemical burns. In contrast, chronic wounds often occur because of endogenous breakdown of the skin caused by systemic diseases such as venous insufficiency, inadequate arterial perfusion, and diabetes. With appropriate topical wound therapy, acute wounds proceed to uncomplicated healing: the initial phase is hemostasis and inflammation, followed by proliferation, epithelialization, and remodeling $[1,2]$. In chronic wounds, a normal repair process becomes disturbed during the phase of inflammation or proliferation [1]. The presence of denatured proteins, cell debris, and fibrin coatings in chronic wounds is a favorable condition for bacterial growth and becomes the cause of impaired wound healing [3]. Not only in chronic but also in acute wounds, bacterial burden contributes to a sustained inflammatory state, which inhibits normal progression to the proliferative phase of healing [1]. Falanga and others developed the concept of wound bed preparation [4]. They introduced a practical and effective method that optimized chronic wound intervention, including resolution of bacterial imbalance $[1,4,5]$. Once the formation of goodquality granulation tissue is achieved through wound preparation, the wound will close either naturally or through skin grafting procedures [6].

Silk-elastins are artificial proteins that were developed by genetic engineering and biological production methods using Escherichia coli [7]. Silk-elastins have a unique feature of temperature-mediated self-gelation [8], which has led to the development of biomaterials using silk-elastin: delivery systems for drugs and viruses [9, 10], 3-dimensional tissue-engineering scaffolds [11], and wound healing materials [12-15]. We have previously shown with in vitro studies and animal models that SE-
$\mathrm{P} 47 \mathrm{~K}$, a type of silk-elastin, promotes wound healing [13-16]. Specifically, the water-soluble form of SE-P47K enhances the migration of fibroblasts and macrophages and induces collagen production in fibroblasts in vitro [16]. Applying SE-P47K solution or sponge to pressure sores of $\mathrm{db} / \mathrm{db}$ mice reduced bacterial growth and promoted granulation tissue formation in comparison with control or carboxymethyl cellulose hydrogels [13, 15].

On the basis of these results, a phase I/II clinical study was conducted, whose main objective was to confirm the safety and feasibility of silk-elastin sponge for treating patients with chronic ulcers [12]. That study was an open-label, single-center study with no control group. No serious adverse events were noted, and the safety of silk-elastin sponge in patients with chronic ulcers was confirmed.

As a next step, to validate the clinical utility of silk-elastin sponge in skin ulcer treatment, a multicenter clinical trial is being conducted.

\section{Objectives}

The central aim of this trial is to evaluate the clinical utility and safety of silk-elastin sponge for the treatment of skin ulcers. In addition, by using a questionnaire to the investigator, the effect of time-saving in the intervention and management will be assessed in comparison with negative pressure wound therapy and treatment with a collagen wound matrix.

\section{Study Design}

This study is a prospective, multicenter, singlearm, uncontrolled clinical trial. The different types of wound, prestudy treatment, interventions, and assessments are summarized in the study design flowchart (Fig. 1) and the Standard Protocol Items: Recommendations for Interventional Trials (SPIRIT) figure [17] (Fig. 2). Participants will be treated with the study device for 14 days and afterward will be observed for 14 days to evaluate the course of wound healing. 


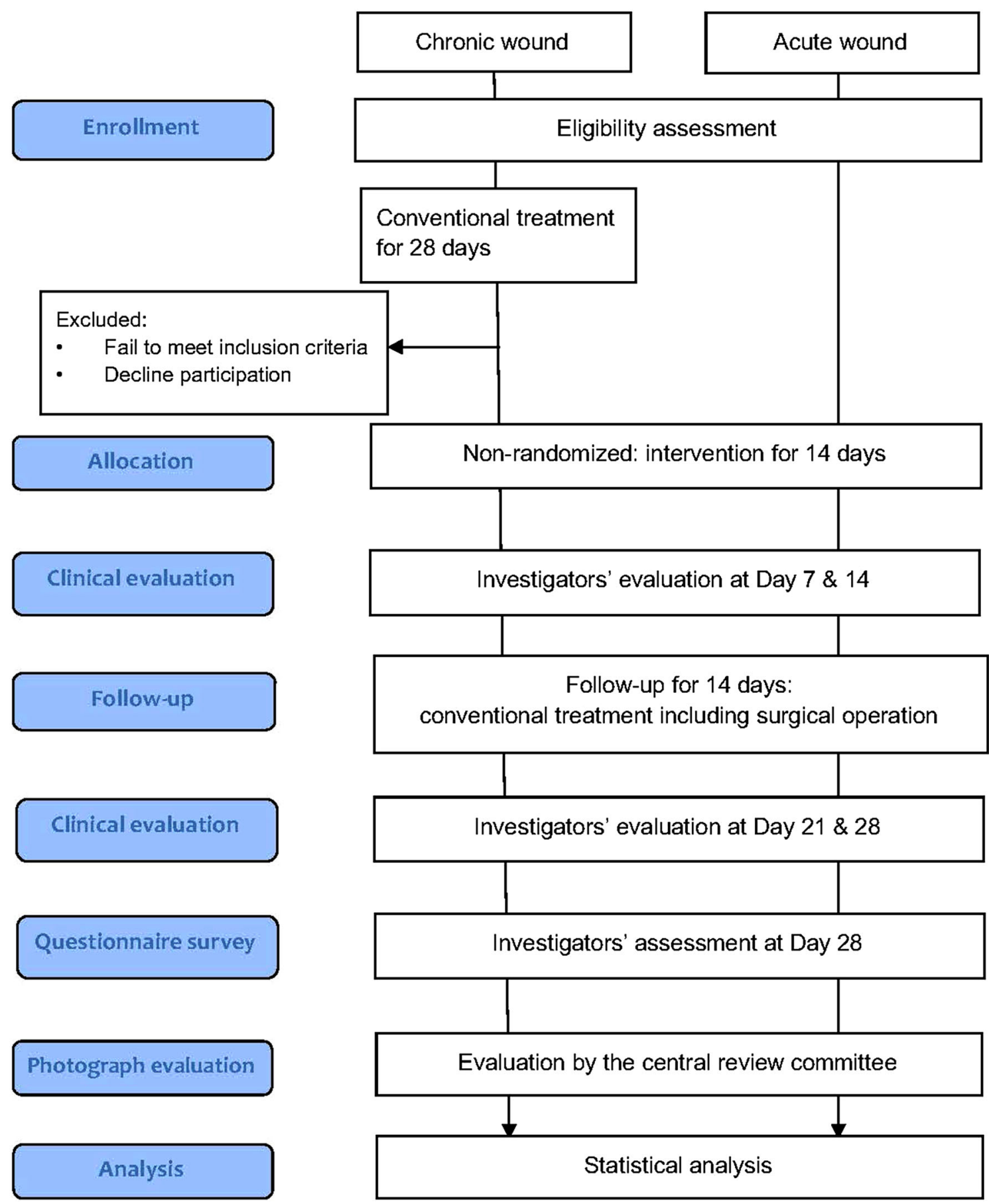

Fig. 1 Study design flowchart. This study is a prospective, multicenter, single-arm, uncontrolled clinical trial. Patients with not acute but chronic wounds will be treated with conventional drugs or materials for 28 days and thereafter rejudged whether they meet inclusion criteria. Participants will be treated with the study device for 14 days and afterward observed for 14 days to evaluate the course of wound healing 


\begin{tabular}{|c|c|c|c|c|c|c|c|c|c|}
\hline & \multicolumn{9}{|c|}{ Study period } \\
\hline & Enrollment & $\begin{array}{c}\text { Conventional } \\
\text { treatment }\end{array}$ & $\begin{array}{c}\text { Before } \\
\text { debridement }\end{array}$ & $\begin{array}{c}\text { After } \\
\text { debridement }\end{array}$ & $\begin{array}{c}\text { Application of } \\
\text { study device }\end{array}$ & $\begin{array}{c}\text { Reapplicati } \\
\text { on }\end{array}$ & Removal & \multicolumn{2}{|c|}{ Follow-up } \\
\hline TIMEPOINT: & & & & & & & & & \\
\hline Chronic wound & & $>28$ days & \multirow{2}{*}{\multicolumn{2}{|c|}{ Day - $\mathbf{1} / \mathbf{0}$}} & \multirow{2}{*}{ Day 0} & \multirow{2}{*}{ Day $7 \pm 2$} & \multirow{2}{*}{ Day 14 \pm 2} & \multirow{2}{*}{ Day $21 \pm 3$} & \multirow{2}{*}{ Day $28 \pm 3$} \\
\hline Acute wound & & None & & & & & & & \\
\hline \multicolumn{10}{|l|}{ ENROLLMENT: } \\
\hline Eligibility screen & 0 & & & & & & & & \\
\hline Informed consent & 0 & & & & & & & & \\
\hline $\begin{array}{r}\begin{array}{r}\text { Confirmation of } \\
\text { eligibility }\end{array} \\
\end{array}$ & & 0 & & & & & & & \\
\hline \multicolumn{10}{|l|}{ INTERVENTIONS: } \\
\hline $\begin{array}{r}\text { Application of } \\
\text { study device }\end{array}$ & & & & & 0 & 0 & & & \\
\hline \multicolumn{10}{|l|}{ ASSESSMENTS: } \\
\hline $\begin{array}{r}\text { Wound size } \\
\text { measurements }\end{array}$ & 0 & 0 & & 0 & & 0 & 0 & 0 & 0 \\
\hline $\begin{array}{r}\text { Photography of } \\
\text { wound }\end{array}$ & 0 & 0 & 0 & 0 & 0 & 0 & 0 & 0 & 0 \\
\hline $\begin{array}{r}\text { Assessm ent of } \\
\text { healthy wound bed }\end{array}$ & & & & & & 0 & 0 & 0 & 0 \\
\hline $\begin{array}{r}\text { Questionnaire } \\
\text { survey }\end{array}$ & & & & & & & & & 0 \\
\hline \multicolumn{10}{|l|}{ Adverse events } \\
\hline $\begin{array}{r}\begin{array}{r}\text { Inflammation- or } \\
\text { infection-related } \\
\text { symptoms }\end{array} \\
\end{array}$ & 0 & 0 & & & & 0 & 0 & 0 & 0 \\
\hline Laboratory tests & 0 & & 0 & & & & 0 & & 0 \\
\hline Vital signs & 0 & & 0 & & 0 & 0 & 0 & & 0 \\
\hline
\end{tabular}

Fig. 2 SPIRIT figure. Schedule of enrollment, interventions, and assessments

\section{METHODS}

\section{Study Setting}

The study is being conducted at five university hospitals in Japan: Kyoto University Hospital (Kyoto), Tokyo Medical University Hospital (Tokyo), Juntendo University Hospital (Tokyo), Kansai Medical University Hirakata Hospital (Osaka), and Kobe University Hospital (Kobe).
The institutional review board of each hospital approved the protocol before patient enrollment.

\section{Inclusion Criteria}

Inclusion criteria for patients with chronic wounds are as follows: (1) The wound area after 28 days of conventional treatment is still more than $50 \%$ of the baseline wound area; (2) The 
wound size (long $\times$ short diameter) after surgical debridement ranges from 2 to $25 \mathrm{~cm}^{2}$; (3) Wound dressing can cover the wound on which the study device is applied; (4) When another wound is located near the wound to be investigated, perilesional skin (the skin between the two wounds) is normal; (5) There is no infection in the wound to be investigated; (6) When the wound involves bone exposure, the proportion of the area with bone exposure is less than 10\% of the wound area; (7) When the ulcer to be investigated is located on the lower extremity, the perfusion pressure of the skin just distal to the ulcer exceeds $30 \mathrm{mmHg}$; and (8) There is an underlying cause that has a reasonable potential to adversely affect healing (e.g., diabetes, venous insufficiency, and pressure sore).

Inclusion criteria for patients with acute wounds are as follows: (1) The following wounds which require wound bed preparation and have no systemic potential to adversely affect healing: complicated wounds, deep second- and third-degree burns, and wounds with limited infection that can be removed by surgical debridement; (2) The wound size (long $\times$ short diameter) after surgical debridement ranges from 2 to $100 \mathrm{~cm}^{2}$; (3) Wound dressing can cover the wound on which the study device is applied; (4) When another wound is located near the wound to be investigated, perilesional skin (the skin between the two wounds) is normal; (5) There is no infection that cannot be removed by surgical debridement; and (6) When the wound involves bone exposure, the proportion of the area with bone exposure is less than $10 \%$ of the wound area.

\section{Exclusion Criteria}

Exclusion criteria for patients with chronic and acute wounds are as follows: (1) The patient is younger than 20 years old when they consent to participate in the trial; (2) Women who are or can be pregnant, who are nursing, and who do not consent to use contraception during the trial; (3) Patients with previous histories of allergy to silk, urethane, and other reagents used in the trial, including local anesthetics and disinfectant; (4) Patients with any of the following conditions: poorly controlled diabetes (10\% HbA1c or higher in the latest laboratory findings within 28 days before enrollment), hypoalbuminemia (less than $2 \mathrm{~g} / \mathrm{dl}$ ), receiving hemodialysis or peritoneal dialysis, and requirement for systemic treatment for malignancy or continuous systemic administration of steroids (a dose exceeding an equivalent prednisolone dose of $10 \mathrm{mg} /$ day); (5) The wound to be investigated is located on a weight-bearing site; (6) Patients who participated in another clinical trial within 3 months before enrollment; (7) Patients who already participated in this trial and received the investigational device; (8) Patients who are not able to consent in writing to participate in the trial; and (9) Patients who are considered by an investigator or sub-investigator to be inappropriate for inclusion in the trial.

\section{Interventions}

\section{Surgical Procedure}

The target ulcer is debrided in the operating room using a scalpel, scissors, and a curette under appropriate anesthesia. After hemostat and irrigation, the study device is applied to the surface of the wound and covered with a wound dressing, which the investigator/sub-investigator chooses from the following: polyurethane film dressing (3M Tegaderm Transparent Film Dressing; 3M, Maplewood, Minnesota, USA), silicone-faced wound dressing (SI-Aid; ALCARE, Tokyo, Japan), low-adherent absorbent dressing (Melolin; Smith \& Nephew, Hull, UK), and polyurethane form dressing (Hydrosite thin type; Smith \& Nephew). If the investigator/subinvestigator is afraid of overproducing wound exudate that causes poor hydrogel formation of silk-elastin, the investigator/sub-investigator applies not the study device but a wound dressing or an ointment. In this case, the investigator/sub-investigator applies the study device on the day after the surgery. Antibiotics are administered intravenously before and after the surgery. 


\section{Reapplication of Study Device}

The investigator/sub-investigator reapplies the study device at 7 days after the initial application of the study device. After a wound dressing is removed and the wound is cleansed with saline, a new study device is applied on the wound, followed by covering with a wound dressing.

If the investigator/sub-investigator observes the formation of a healthy granulation on the scheduled day of reapplication, the investigator/sub-investigator can withdraw the reapplication of the study device.

\section{Duration of Treatment, Subsequent Therapy, and Follow-up Period}

The treatment ends 14 days after the initial application of the study device. The investigator/sub-investigator resumes conventional treatment for the wound after the removal of the study device. The conventional treatment includes conservative therapy and simple surgical procedures (e.g., direct suturing, skin grafting, and reconstruction with a local skin flap) but not negative pressure wound therapy. The observation of the wound is performed 28 days after the initial application of the study device.

\section{Discontinuation Criteria}

Discontinuation criteria are as follows: (1) The patient's physical condition is deteriorated by any cause; (2) Between the day of enrollment and surgical debridement, the wound develops acute exacerbation and does not satisfy the eligibility criteria; (3) After the surgical debridement, the wound area exceeds $25 \mathrm{~cm}^{2}$ (chronic wound) or $100 \mathrm{~cm}^{2}$ (acute wound); (4) Allergic symptoms do not decline in spite of appropriate therapy; (5) Wound infection is observed after the application of the study device; (6) A serious adverse event is observed during the treatment with the study device; (7) The patient requests discontinuation of the study; (8) The entire study is discontinued; and (9) The investigator/sub-investigator considers it inappropriate to continue the study for any other reason.

\section{Endpoints}

\section{Clinical Utility Endpoints}

The primary endpoint is the frequency of patients with chronic wounds in whom the investigator/sub-investigator confirms the formation of a healthy wound bed at 14 days after the initial application of the study device. The formation of a healthy wound bed satisfies the following three criteria: a healthy granulation area exceeds $80 \%$ of the wound area; a nonviable tissue area does not exceed $5 \%$ of the wound area; and there is no wound infection.

The secondary endpoints are as follows: (1) frequency of patients in whom the investigator/sub-investigator confirms the formation of a healthy wound bed at 7,14 (acute wound only), 21 , and 28 days after the initial application of the study device; (2) ratio of wound area (wound area/baseline wound area) at 7, 14, 21, and 28 days after the initial application of the study device; (3) proportion of healthy granulation area (healthy granulation area/wound area) at $7,14,21$, and 28 days after the initial application of the study device; (4) frequency of the application of the study device to each patient, (5) questionnaire survey of the investigators/sub-investigators to assess the handiness of the study device.

\section{Safety Endpoints}

The safety endpoints are as follows: (1) incidence of adverse events, which are defined as any untoward medical occurrence in a participant that does not necessarily have a causal relationship with the trial intervention, (2) incidence of inflammation- or infection-related symptoms (redness, heat, swelling, pain exacerbation, increased exudate, cloudy exudate, and malodor) at 7 and 14 days after the initial application of the study device.

\section{Study Schedule}

After confirmation that a patient is to be initiated on silk-elastin sponge treatment, they will be enrolled by the start of conventional 
treatment (chronic wound) or by the scheduled day of debridement (acute wound). For patients with chronic wounds, the investigator/sub-investigator will evaluate the wound area after 28-day conventional treatment. Only for patients whose wound area is greater than $50 \%$ of the wound area before the conventional treatment, the investigator/sub-investigator will perform debridement and application of the study device.

\section{Evaluation of Healthy Wound Bed, Wound Area, and Healthy Granulation Area}

\section{Investigator/Sub-investigator}

The investigator/sub-investigator will photograph the chronic wound before and after 28-day conventional treatment and measure the wound area using digital images. On the other hand, the investigator/sub-investigator will assess at the bedside whether the wound satisfies the following three criteria: a healthy granulation area exceeds $80 \%$ of the wound area; a nonviable tissue area does not exceed 5\% of the wound area; and there is no wound infection.

\section{Central Review Committee}

The central review committee will measure the ratio of wound area and the proportion of healthy granulation area using digital images. In addition, the formation of a healthy wound bed, which is assessed by the investigator/subinvestigator at the bedside, will be checked by the central review committee using digital images.

\section{Digital Photography of Wounds}

The digital images of the wounds are taken with a calibrator (Casmatch; Bear Medic, Tokyo, Japan). Their color and size are calibrated according to the manufacturer's instructions, and the size of the area is measured using a raster graphics editor (Adobe Photoshop; Adobe, San Jose, California, USA).

\section{Sample Size}

The target sample size for this trial is 20 for patients with chronic wounds. This number is based on the results of previous clinical trials. In the previous study of silk-elastin sponge in patients with chronic wounds in the lower leg, 4 out of 6 patients $(67 \%)$ showed the formation of a healthy wound bed at 5-16 days after the application of silk-elastin sponge [12]. For comparison, a clinical trial in Japan using a negative pressure wound therapy device (Renasys; Smith \& Nephew) for chronic and acute wounds is a reasonable reference because the endpoints of the Renasys trial are similar to those of this silk-elastin sponge trial [18]. In the Renasys trial, the frequency of patients with chronic wounds who demonstrated the formation of a healthy wound bed after 14-day treatment was estimated to be $34 \%$ [18]. The minimal sample size required to have a power of greater than $80 \%$ in a two-sided test at a significance level of $5 \%$ is estimated to be 17 patients. Considering possible dropouts, the number of patients is set at 20 .

The target sample size for patients with acute wounds is defined as 5 . To assess the safety of the study device for the treatment of acute wounds with intractable properties such as complicated wounds, deep second- and thirddegree burns, and wounds with limited infection that can be removed by debridement, only a small number of patients will be included.

\section{Statistical Analyses}

\section{Analysis Set}

Full analysis set (FAS): population of patients in whom the study device has been applied at least once and have provided the data necessary to evaluate the primary efficacy endpoint.

Per protocol set (PPS): population of patients included in the FAS who have no protocol violation.

Safety analysis set (SAS): population of patients in whom the study device has been applied at least once. 


\section{Clinical Utility Analyses}

Clinical utility analyses will be performed in the FAS and PPS.

Analysis of the primary endpoint is done by calculating 95\% confidence interval (CI) of the frequency of patients with chronic wounds in whom the investigator/sub-investigator confirms the formation of a healthy wound bed at 14 days after the initial application of the study device.

Analyses of the secondary endpoints of chronic wounds are as follows: (1) calculation of $95 \%$ CI of the frequency of patients in whom the investigator/sub-investigator confirms the formation of a healthy wound bed at 7,21, and 28 days after the initial application of the study device; (2) calculation of $95 \%$ CI of the ratio of wound area (wound area/baseline wound area) at $7,14,21$, and 28 days after the initial application of the study device; (3) calculation of $95 \%$ CI of the proportion of healthy granulation area (healthy granulation area/wound area) at $7,14,21$, and 28 days after the initial application of the study device; (4) calculation of summary statistics of the frequency of the application of the study device to each patient; and (5) tabulation of the assessment results in a questionnaire survey on the handiness of the study device.

Analyses of the secondary endpoints of acute wounds are as follows: (1) calculation of $95 \% \mathrm{CI}$ of the frequency of patients in whom the investigator/sub-investigator confirms the formation of a healthy wound bed at 7, 14, 21, and 28 days after the initial application of the study device; (2) the ratio of wound area (wound area/ baseline wound area) at 7, 14, 21, and 28 days after the initial application of the study device; (3) the proportion of healthy granulation area (healthy granulation area/wound area) at 7, 14, 21 , and 28 days after the initial application of the study device; (4) the frequency of the application of the study device to each patient; and (5) tabulation of the assessment results in a questionnaire survey on the handiness of the study device.

The calculation of $95 \%$ CI will be performed by Base SAS 9.4 using the F distribution.

\section{Safety Analyses}

Safety analyses will be performed in the SAS.

Analyses of the safety endpoints are as follows: (1) incidence of adverse events; (2) tabulation of serious adverse events; (3) calculation of summary statistics of the laboratory data (white blood cell count, red blood cell count, hemoglobin, hematocrit, platelet count, total protein, albumin, total bilirubin, aspartate aminotransferase, alanine aminotransferase, $\gamma$ glutamyl transpeptidase, creatinine, urea nitrogen, and C-reactive protein) at four points as follows: before enrollment, before debridement, and 14 and 28 days after the application of the study device; and (4) calculation of summary statistics of the vital signs (body temperature, blood pressure, and heart rate) at six points as follows: before enrollment, before debridement, immediately after the application of the study device, and 7, 14, and 28 days after the application of the study device.

\section{ETHICS AND DISSEMINATION}

The study is performed in compliance with the ethical principles of the 1975 Declaration of Helsinki, the Act of Japan on Securing Quality, Efficacy, and Safety of Products Including Pharmaceuticals and Medical Devices, and the Ministerial Ordinance of Good Clinical Practice. The study protocol was approved by the institutional review board (IRB) of each institution: Kyoto University Certified Review Board; IRB of Tokyo Medical University Hospital; IRB of Juntendo University Hospital; IRB of Kansai Medical University Hospital; and Kobe University Clinical Research Ethical Committee. The Japanese regulatory agency, Pharmaceuticals Medical Devices Agency (PMDA), was notified regarding this medical-device clinical trial on 14 April 2021. All patients will be required to provide written informed consent.

Results of this trial will be submitted to an international peer-reviewed journal after conclusion of the trial by the sponsor-investigator. 


\section{DISCUSSION}

This multicenter, single-arm clinical trial to evaluate the clinical utility of silk-elastin sponge for skin ulcers is conducted to gain approval from PMDA in Japan. The efficacy of a negative pressure wound therapy device for diabetic foot ulcers is well established [19-21]. In Japan, a clinical trial to evaluate the clinical utility of Renasys was conducted previously [18]. In that uncontrolled trial of Renasys, the endpoint was the time until the wound developed a healthy wound bed, in which the wound satisfied the following three criteria: a healthy granulation area exceeded $80 \%$ of the wound area; nonviable tissue area did not exceed 5\% of the wound area; and there was no infection of the wound. Identically to the design of the Renasys trial [18], this trial of silk-elastin sponge was designed as a single-arm, uncontrolled trial with similar endpoints.

The results of the previous clinical study of silk-elastin sponge revealed that poor hydrogel formation, possibly due to excessive exudate, caused unhealthy granulation tissue formation [12]. In this trial, to reduce the exudate and promote hydrogel formation, the investigator can use wound dressing that absorbs the exudate or apply the silk-elastin sponge on the next day of debridement.

We believe that this trial will demonstrate the clinical utility of silk-elastin sponge for the treatment of chronic and acute ulcers that are difficult to treat.

\section{STRENGTHS AND LIMITATIONS}

A novel wound-healing material for the treatment of chronic skin ulcers is much needed. On the basis of the historical data on an approved medical device, this study was designed as a single-arm trial. This trial intends to treat not only chronic but also acute wounds. However, this is not a large-scale study.

\section{ACKNOWLEDGEMENTS}

The authors thank Shingo Kawabata (Sanyo Chemical), the person in charge of this trial, for preparation of the material and supporting this trial.

Funding. This study is supported by the AMED Acceleration Transformative Research for Medical Innovation (ACT-M) program (Grant No. JP20he1302037), and partially supported by Sanyo Chemical. The journal's Rapid Service Fee will be paid from the AMED grant.

Editorial Assistance. The authors thank Miho Kobayashi for editing the manuscript. Ms. Kobayashi is a full-time professional English editor at Kurashiki Central Hospital and paid by the hospital manager.

Authorship. All named authors meet the International Committee of Medical Journal Editors (ICMJE) criteria for authorship for this article. All authors approved the final manuscript.

Authors Contributions. KN, YK, ES, MS, and NM designed the protocol with SK. HY, IT, and MK will conduct the trial. KN prepared the first draft of the manuscript.

Disclosures. Kazuo Noda, Yasuhiro Katayama, Eiichi Sawaragi, Michiharu Sakamoto, Hiroki Yamanaka, Itaru Tsuge, Motoki Katsube, and Naoki Morimoto have nothing to disclose. Sanyo Chemical had no role in decision to publish, or preparation of the manuscript.

Compliance with Ethics Guidelines. The study is performed in compliance with the ethical principles of the 1975 Declaration of Helsinki, the Act of Japan on Securing Quality, Efficacy, and Safety of Products Including Pharmaceuticals and Medical Devices, and the Ministerial Ordinance of Good Clinical Practice. 
The study protocol was approved by the institutional review board (IRB) of each institution: Kyoto University Certified Review Board; IRB of Tokyo Medical University Hospital; IRB of Juntendo University Hospital; IRB of Kansai Medical University Hospital; and Kobe University Clinical Research Ethical Committee. The Japanese regulatory agency, Pharmaceuticals Medical Devices Agency (PMDA), was notified regarding this medical-device clinical trial on 14 April 2021. All patients will be required to provide written informed consent.

Open Access. This article is licensed under a Creative Commons Attribution-NonCommercial 4.0 International License, which permits any non-commercial use, sharing, adaptation, distribution and reproduction in any medium or format, as long as you give appropriate credit to the original author(s) and the source, provide a link to the Creative Commons licence, and indicate if changes were made. The images or other third party material in this article are included in the article's Creative Commons licence, unless indicated otherwise in a credit line to the material. If material is not included in the article's Creative Commons licence and your intended use is not permitted by statutory regulation or exceeds the permitted use, you will need to obtain permission directly from the copyright holder. To view a copy of this licence, visit http:// creativecommons.org/licenses/by-nc/4.0/.

\section{REFERENCES}

1. Schultz GS, Sibbald RG, Falanga V, et al. Wound bed preparation: a systematic approach to wound management. Wound Repair Regen. 2003;11(Suppl 1):S1-28.

2. Singer AJ, Clark RA. Cutaneous wound healing. N Engl J Med. 1999;341(10):738-46.

3. Kaehn K, Eberlein T. In-vitro test for comparing the efficacy of wound rinsing solutions. Br J Nurs. 2009;18(11):S4,S6-S8, S10.

4. Falanga V. Classifications for wound bed preparation and stimulation of chronic wounds. Wound Repair Regen. 2000;8(5):347-52.
5. Granick M, Boykin J, Gamelli R, Schultz G, Tenenhaus M. Toward a common language: surgical wound bed preparation and debridement. Wound Repair Regen. 2006;14(Suppl 1):S1-10.

6. Fonder MA, Lazarus GS, Cowan DA, Aronson-Cook B, Kohli AR, Mamelak AJ. Treating the chronic wound: a practical approach to the care of nonhealing wounds and wound care dressings. J Am Acad Dermatol. 2008;58(2):185-206.

7. Cappello J, Crissman J, Dorman M, et al. Genetic engineering of structural protein polymers. Biotechnol Prog. 1990;6(3):198-202.

8. Cappello J, Crissman JW, Crissman M, et al. In-situ self-assembling protein polymer gel systems for administration, delivery, and release of drugs. J Control Release. 1998;53(1-3):105-17.

9. Hatefi A, Cappello J, Ghandehari H. Adenoviral gene delivery to solid tumors by recombinant silkelastinlike protein polymers. Pharm Res. 2007;24(4):773-9.

10. Greish K, Frandsen J, Scharff S, et al. Silk-elastinlike protein polymers improve the efficacy of adenovirus thymidine kinase enzyme prodrug therapy of head and neck tumors. J Gene Med. 2010;12(7): $572-9$.

11. Qiu W, Huang Y, Teng W, Cohn CM, Cappello J, Wu X. Complete recombinant silk-elastinlike protein-based tissue scaffold. Biomacromol. 2010;11(12):3219-27.

12. Noda K, Kawai K, Matsuura Y, et al. Safety of silkelastin sponges in patients with chronic skin ulcers: a phase I/II, single-center, open-label, single-arm clinical trial. Plast Reconstr Surg Glob Open. 2021;9(4):e3556.

13. Kawai K, Kanda N, Kawabata S, Suzuki S. The effect of silk-elastin in the pressure ulcers. Jpn J PU. 2013;15(1):41-7.

14. Kawabata S, Kanda N, Hirasawa Y, et al. The utility of silk-elastin hydrogel as a new material for wound healing. Plast Reconstr Surg Glob Open. 2018;6(5): e1778.

15. Kawabata S, Kawai K, Somamoto S, et al. The development of a novel wound healing material, silk-elastin sponge. J Biomater Sci Polym Ed. 2017;28(18):2143-53.

16. Ozaki C, Somamoto S, Kawabata S, Tabata Y. Effect of an artificial silk elastin-like protein on the migration and collagen production of mouse fibroblasts. J Biomater Sci Polym Ed. 2014;25(12): 1266-77. 